\title{
基于卷积神经网络的红外与可见光图像融合
}

\author{
董安勇 ${ }^{1}$ ，杜庆治 ${ }^{1}$ ，苏 斌 $^{2}$ ，赵文博 ${ }^{2}$ ，于 闻 ${ }^{2}$
}

(1. 昆明理工大学 信息工程与自动化学院, 云南 昆明 650500; 2. 昆明北方红外技术股份有限公司, 云南 昆明 650500)

\begin{abstract}
摘要: 非下采样剪切波变换（NSST）域中低频子带的融合需要人工给定融合模式，因此未能充分捕 获源图像的空间连续性和轮廓细节信息。针对上述问题, 提出了基于深度卷积神经网络的红外与可见 光图像融合算法。首先, 使用孪生双通道卷积神经网络学习NSST 域低频子带的特征来输出衡量子带 空间细节信息的特征图。然后，根据高斯滤波处理的特征图设计了基于局部相似性的测量函数来自适 应地调整 NSST 域低频子带的融合模式。最后, 根据 NSST 域高频子带的方差、局部区域能量以及可 见度特征来自适应地设置脉冲耦合神经网络参数完成 NSST 域高频子带的融合。实验结果表明: 该算 法 $Q^{\mathrm{AB} / \mathrm{F}}$ 指标略弱于对比算法，但 SF、SP、SSIM 以及 VIFF 指标分别提高了约 $50.42 \% 、 14.25 \% 、 7.91 \%$ 以及 $61.67 \%$, 有效地解决了低频子带融合模式给定的问题, 同时又克服了手动设置 PCNN 参数的缺 陷。
\end{abstract}

关键词: 图像融合; 卷积神经网络; 参数自适应脉冲耦合神经网络; NSST 变换

中图分类号：TP751.1 文献标识码：A 文章编号：1001-8891(2020)07-0660-10

\section{Infrared and Visible Image Fusion Based on Convolutional Neural Network}

\author{
DONG Anyong ${ }^{1}$, DU Qingzhi ${ }^{1}$, SU Bin ${ }^{2}$, ZHAO Wenbo ${ }^{2}$, YU Wen ${ }^{2}$ \\ (1. Kunming University of Science and Technology, Faculty of Information Engineering and Automation, Kunming 650500, China;
}

2. Kunming North Infrared Technology Co., Ltd, Kunming 650500, China)

\begin{abstract}
The fusion of the low-frequency subband in the non-subsampled shearlet transform (NSST) domain requires artificially obtained fusion modes; thus, the spatial continuity and contour detail information of the source image are not adequately captured. An infrared and visible image fusion algorithm based on a convolutional neural network is proposed to solve this problem. First, the Siamese convolutional neural network is used to learn the characteristics of the low-frequency subband in the NSST domain and output a feature map that measures the spatial detail information of the subbands. Then, on the basis of the feature map obtained by Gaussian filter processing, a local-similarity-based measurement function is designed to adaptively adjust the fusion mode of the low-frequency subband in the NSST domain. Finally, on the basis of the variance of the high-frequency subband in the NSST domain, the local region energy, and the visibility characteristics, the pulse-coupled neural network (PCNN) parameters are adaptively set to complete the fusion of the high-frequency subband in the NSST domain. Experimental results show that the $Q^{\mathrm{AB} / \mathrm{F}}$ index of the algorithm is slightly lower than that of the comparison algorithm. However, the spatial frequency, SP, structural similarity, and visual information fidelity for fusion are improved by approximately $50.42 \%, 14.25 \%, 7.91 \%$, and $61.67 \%$, respectively, which indicates that the method effectively solves the low-frequency subband fusion mode. It also eliminates the need to manually set the PCNN parameters to solve this problem.
\end{abstract}

Key words: image fusion, convolutional neural network, parameter adaptive pulse coupled neural network, NSST transform 


\section{0 引言}

红外成像传感器具有捕捉物体热辐射的能力, 但 对场景纹理细节信息的变化不具有敏感性。可见光成 像传感器具有捕捉场景空间细节信息的能力, 但容易 遭受周围恶劣环境的影响。因此, 融合红外与可见光 图像可以捕捉各波段互补特征, 显示辐射目标保留场 景信息。目前, 在情报收集、监控安全以及航空航天 等领域有广泛的应用前景。

非下采样剪切波变换 (non-subsampled shearlet transform, NSST) 具有平移不变特性和稀疏表示能力, 在图像融合领域已经成为众多学者研究的热点。文献 [1]提出了稀疏表示和非下采样 Shearlet 变换相结合的 多聚焦图像融合算法, 该算法可以使用融合的低频子 图构造过完备字典, 捕捉源图像更多的边缘、纹理以 及细节信息。文献[2]提出了 NSST 与引导滤波相结合 的多聚焦图像融合算法, 该算法设计了基于局部区域 显著性特征的引导滤波加权融合模式, 可以保留源图 像较多的对比度和清晰度信息。但是, 上述算法的融 合模式都是人为给定的, 缺乏自适应调节的能力。

卷积神经网络 (convolutional neural network, CNN) 可以将数据特征进行分层表示, 从而有效地挖 掘数据局部和全局的特征信息, 可以克服现有算法缺 乏学习自我特征能力的缺陷。文献[3]提出了基于卷积 神经网络的红外与可见光图像融合算法, 该算法使用 卷积神经网络得到了衡量源图像特征信息的决策图 来自适应调整融合模式。文献[4]提出了基于图像分割 的卷积神经网络多聚焦图像融合算法, 该算法通过卷 积神经网络将源图像进行分割来输出对应的决策图。 但是, 上述算法没有从多尺度角度将源图像划分为高 低频子带进行处理，不符合人眼视觉系统的特性。

脉冲耦合神经网络 (pulse coupled neural network, PCNN）模型具有全局耦合和脉冲同步的特性, 可以 将变换域高频子带融入丰富的视觉特性信息。文献[5] 提出了基于自适应 PCNN 模型的四元数小波域图像 融合算法, 该算法使用拉普拉斯算子优化 PCNN 模型 的阈值, 改善了融合图像的视觉效果。但是, 该算法 未能解决人工设定自由参数的困难, 不利于自适应地 调整网络参数。文献[6]提出参数自动设置的简易 PCNN 模型, 该模型可以根据图像的特征信息自适应 地设置 PCNN 模型的自由参数, 具有较强的适用性。

针对上述问题分析, 本文综合 NSST 变换、CNN （convolutional neural network）模型以及 PCNN 模型 的互补特性, 提出了基于深度卷积神经网络的红外与 可见光图像融合算法 (NSST-CNN-APCNN (Adaptive
Pulse Coupled Neural Network, APCNN）算法）。该 算法从变换域角度解决了低频子带不具有融合模式 自适应调整的能力, 同时又避免了经典简易 PCNN 模 型手动设置自由参数的困难, 增强了算法的表现力, 优化了融合处理过程, 具有较高的适用性。

\section{NSST 变换与 PCNN 模型}

\section{1 非下采样剪切波变换}

NSST 变换主要包括两个处理过程, 即多尺度分 解和方向局部化。多尺度分解过程是使用金字塔滤波 器 (non-subsampled pyramid filter, NSPF) 将源图像分 解成 $k+1$ 个子带 (1 个低频分量和 $k$ 个高频分量)。 方向局部化过程使用剪切波滤波器将高频分量进行 $j$ 级方向变换, 可以得到 $2^{j}$ 个高频分量, 很大程度上克 服了伪吉布斯效应 ${ }^{[7]}$ 。“store” 图像 2 级 NSST 变换 过程如图 1 所示, 分解的部分子带如图 2 所示 (其中, (a)为低频子带，(b)和(c)为高频子带)。

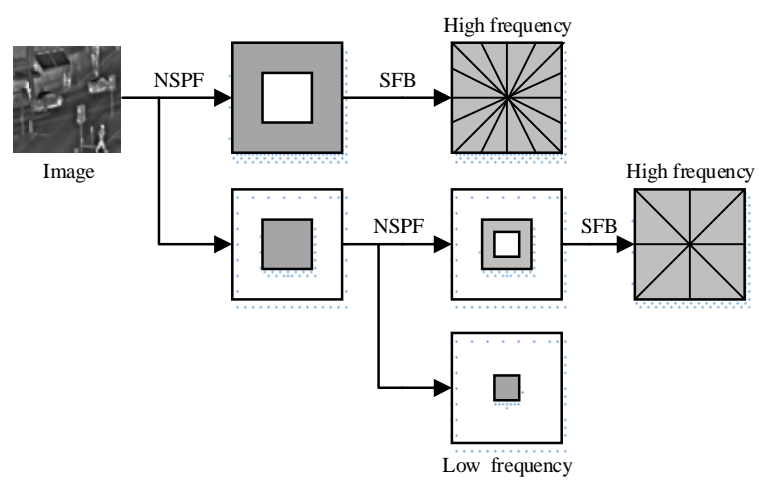

图 1 NSST 变换过程Ｆig.1 NSST transformation process

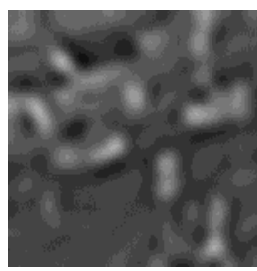

(a) 低频子带

(a) Low frequency subband

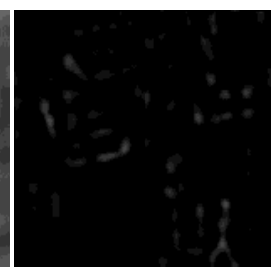

(b) 高频子带 1

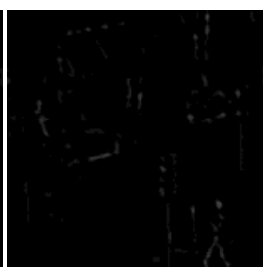

(c) 高频子带 2 $\begin{array}{ll}\text { (b) High frequency } & \text { (c) High frequency }\end{array}$ subband 1

图 2 NSST 分解子带 Fig.2 NSST decomposition subband

\section{2 参数自适应脉冲耦合神经网络}

为了解决经典 PCNN 模型需要人为设置自由参 数的问题 ${ }^{[8-9]}$, 本文选用了参考文献[10]提出的参数自 适应脉冲耦合神经网络 (简称为 APCNN 模型)。 APCNN 模型的数学描述如式(1): 


$$
\left\{\begin{array}{l}
F_{i j}[n]=S_{i j} \\
L_{i j}[n]=V_{\mathrm{L}} \sum_{k l} W_{i j k l} Y_{k l}[n-1] \\
U_{i j}[n]=\mathrm{e}^{-\alpha_{\mathrm{L}}} U_{i j}[n-1]+F_{i j}[n]\left(1+\beta L_{i j}[n]\right) \\
E_{i j}[n]=\mathrm{e}^{-\alpha_{E}} E_{i j}[n-1]+V_{E} Y_{i j}[n] \\
Y_{i j}[n]=\left\{\begin{array}{l}
1, U_{i j}[n]>E_{i j}[n] \\
0, \text { otherwise }
\end{array}\right.
\end{array}\right.
$$

在上述 APCNN 模型中, $F_{i j}[n] 、 L_{i j}[n] 、 U_{i j}[n]$ 以 及 $E_{i j}[n]$ 分别为 $(i, j)$ 坐标神经元迭代 $n$ 次的反馈输入、 链接输入、内部活动以及动态阈值。 $V_{\mathrm{L}}$ 和 $\alpha_{\mathrm{L}}$ 分别为 链接输入的幅度系数和时间常数。 $V_{E}$ 和 $\alpha_{E}$ 分别为变 阈值函数的幅度系数和时间常数。 $S_{i j}, \beta$ 以及 $W_{i j k l}$ 分 别为输入激励、链接强度以及突触连接系数。从 APCNN 模型中可以看出, 存在 $V_{\mathrm{L}} 、 V_{E} 、 \alpha_{\mathrm{L}} 、 \alpha_{E}$ 以及 $\beta 5$ 个自由参数。其中, $V_{\mathrm{L}}$ 和 $\beta$ 仅为 $\sum_{k l} W_{i j k l} Y_{k l}[n-1]$ 的

权重。因此, 设 $\lambda=\beta V_{\mathrm{L}}$ 表示加权链接强度, 将 $\mathrm{APCNN}$ 模型用来处理图像融合问题, 可以自适应地给出剩余 4 个自由参数的数学描述:

$$
\begin{gathered}
\alpha_{\mathrm{L}}=\lg (1 / \sigma(S)) \\
\lambda=\frac{\left(S_{\max } / S^{\prime}\right)-1}{6} \\
V_{E}=\mathrm{e}^{-\alpha_{\mathrm{L}}}+1+6 \lambda \\
\alpha_{E}=\ln \left(\frac{V_{E} / S^{\prime}}{\frac{1-\mathrm{e}^{-3 \alpha_{\mathrm{L}}}}{1-\mathrm{e}^{-\alpha_{\mathrm{L}}}}+6 \lambda \mathrm{e}^{-\alpha_{\mathrm{L}}}}\right)
\end{gathered}
$$

式中: $\sigma(S)$ 为输入图像的标准偏差; $S^{\prime}$ 和 $S_{\text {max }}$ 分别表 示归一化阈值和输入图像的最大强度。

\section{2 卷积神经网络}

卷积神经网络可以利用高维空间关系来解决人 为设定特征信息的问题, 从而具有很强的特征学习和 特征提取能力。CNN 模型主要由输入层、卷积层、池 化层、全连接层以及输出层组成 ${ }^{[11]}, \mathrm{CNN}$ 模型的基 本结构如图 3 所示。其中, 卷积层是模型的核心部分, 主要作用是提取数据特征。每个卷积核都可以提取上
一层输入数据的特征, 且随着卷积核数量的不同, 提 取的特征丰富度也不同。池化层位于卷积层之后, 主 要作用是为了优化参数规模、降低空间容量以及减少 过拟合的影响。全连接层位于模型的末端, 类似于人 工模型的全连接方式。另外, 如果需要解决分类问题, 可以依次连接分类层。

对于基于 $\mathrm{CNN}$ 模型的图像融合算法来说, 图像 融合的处理转变成了 CNN 模型的设计, 融合模式可 以通过训练 CNN 模型自动生成。同时, 学习的特征 数据可以被视为 “最优” 方案。因此, 基于 CNN 模 型的融合算法可以有效地解决需要人为设定自由参 数的难题。为了保证两幅源图像特征提取和信息量估 计的处理方式是一致的, 本文选用了参考文献[12]设 计的孪生双通道神经网络 (siamese convolution neural network, SCNN)。SCNN 模型可以通过双通道的 CNN 特征学习将双通道输入图像的匹配度进行估计, 并由 分类器输出图像匹配的结果, 具有较高的收玫性。

\section{3 基于 NSST-CNN-APCNN 的红外与可见光 图像融合算法}

NSST-CNN-APCNN 算法首先将红外与可见光图 像进行 4 级 NSST 分解得到具有结构特征的低频子带 和保留细节特征的高频子带。然后, 将 NSST 域低频 子带输送到孪生双通道 CNN 模型, 经过 CNN 模型的 特征学习和分类来评估出决策图, 再根据决策图输出 概率值和局部相似性测量函数, 判别出可以自适应调 整的融合模式来完成 NSST 域低频子带的融合。其次, 使用 NSST 域高频子带的绝对值作为 APCNN 模型的 激励输入, 同时分别使用方差、区域能量以及可见度 特征信息来调节式(2)中 $\sigma(S)$ 的值。因此, 可以根据输 入特征自适应地调整 APCNN 模型的自由参数, 从而 输出点火映射图来完成 NSST 域高频子带的融合。最 后, 通过使用 NSST 逆变换将红外与可见光融合图像 进行重构。基于 NSST-CNN-APCNN 的红外与可见光 图像融合算法框架如图 4 所示。

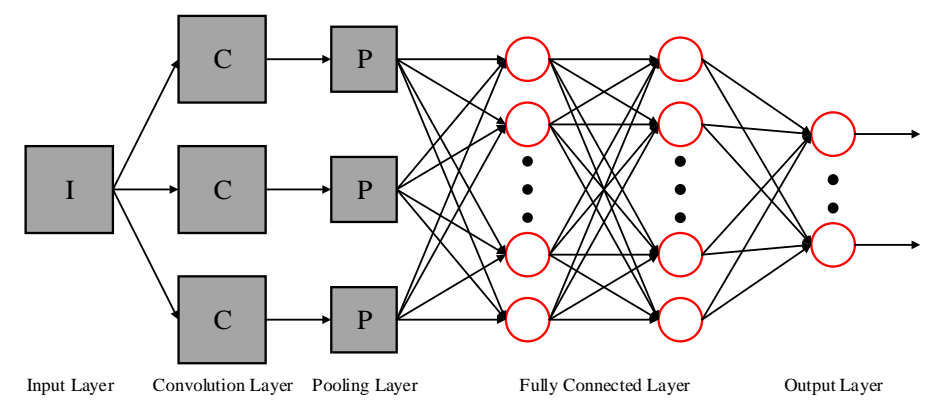

图 $3 \mathrm{CNN}$ 模型基本结构

Fig.3 Basic structure of the CNN model 


\section{1 基于 SCNN 模型的低频系数融合}

NSST 域低频子带系数融合决策图的生成可以被 认为是分类问题的处理。具体来说, 可以通过测量图 像块信息量的多少得到系数融合的决策图。SCNN 模 型的网络结构具有相同权重和相同体系的两个输入 分支, 因此 SCNN 模型对于两个输入图像块的特征提 取和信息估计方式是一致的, 且容易被数据集训练, 具有较好的收玫性。本文选用的 SCNN 模型基本结构 如图 5 所示。

本文算法将 NSST 域低频子带分别输送到 SCNN 模型的两个分支, 通过 3 个卷积层和一个最大池化层 可以得到两个衡量图像块信息量的特征映射。两个特
征映射通过神经元数目分别为 256 和 2 的两个全连接 层实现决策分配可以得到最终的概率值决策图。其 中, 神经元数目为 2 的全连接层是分类层, 神经元数 目 2 为分类类别, 通过分类器 Softmax 可以输出分类 的概率值。这样, SCNN 模型输出的决策图决定了对 应位置处源图像块细节信息的多少。为了使人类视觉 神经系统获得更好的感知效果, 本文算法首先将 NSST 域低频子带进行了拉普拉斯分解, 然后将 SCNN 模型输出的决策图进行了高斯滤波分解，最后在每个 分解层级上完成 NSST 域低频子带系数的融合。NSST 域低频子带系数融合的基本框架如图 6 所示，具体的 融合流程如下所示。

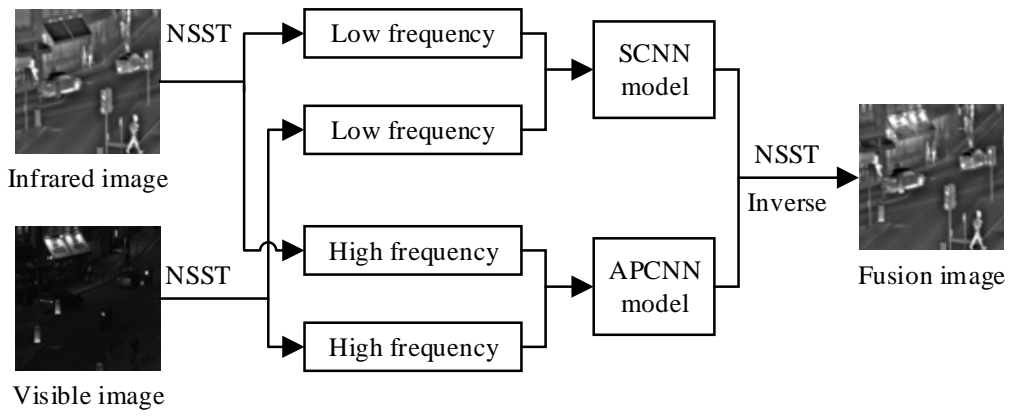

图 4 基于 NSST-CNN-APCNN 的图像融合框架 Fig.4 Image fusion framework based on NSST-CNN-APCNN

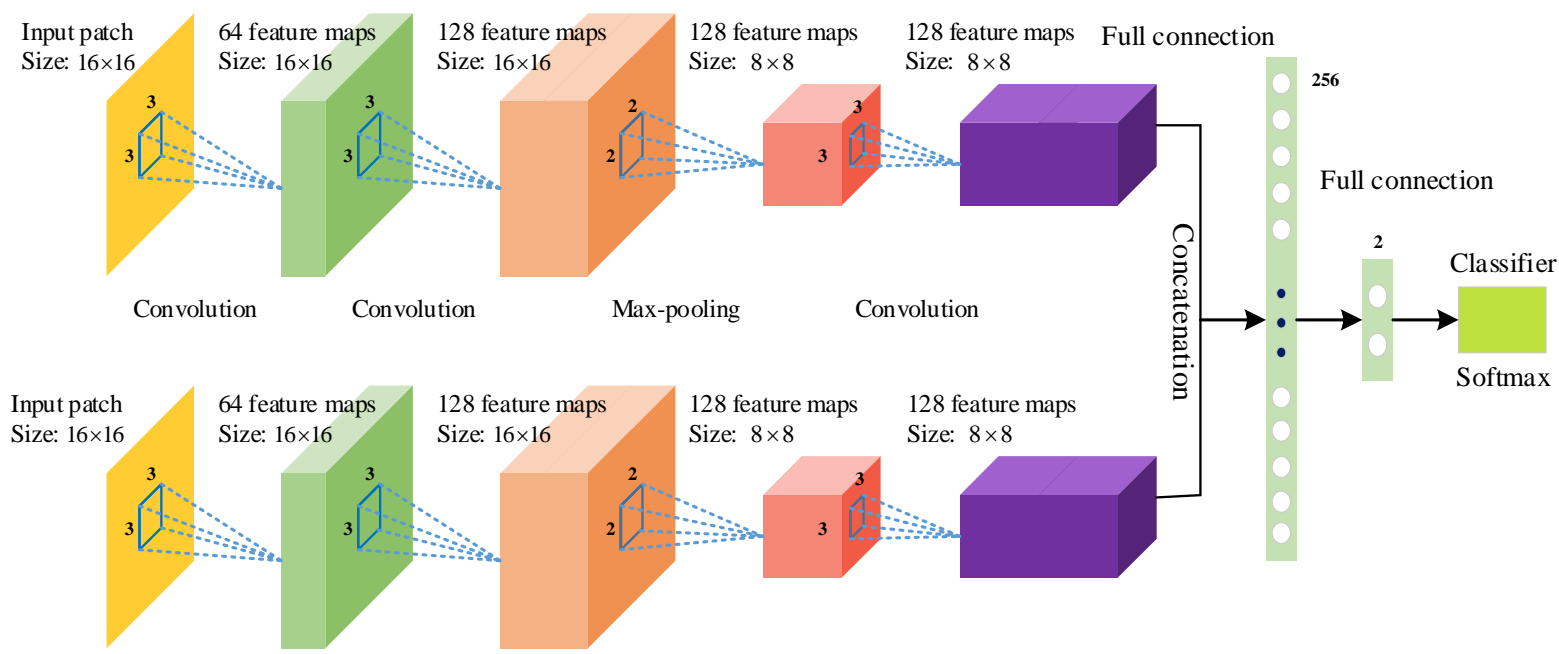

图 5 SCNN 模型基本结构 Fig.5 Basic structure of SCNN model

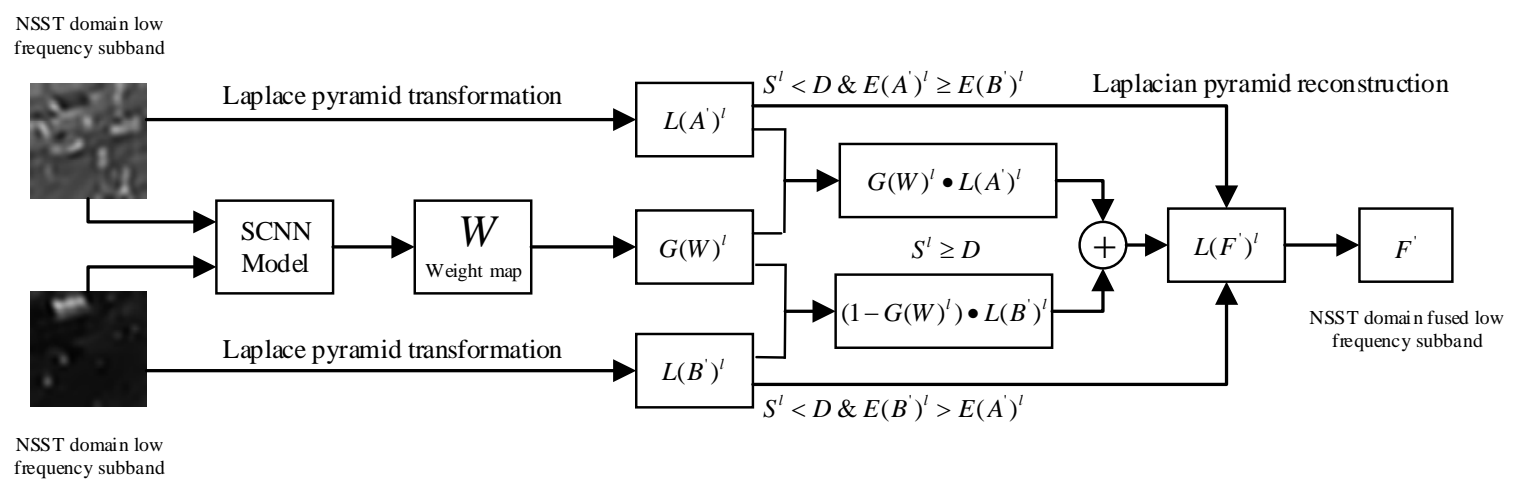

图 6 低频子带系数融合基本框架

Fig.6 Basic framework for the fusion of low-frequency subband coefficients 
步骤 1: 将大小为 $256 \times 256$ 的红外低频子图 $A_{\mathrm{L}}$ 和可见光低频子图 $B_{\mathrm{L}}$ 分成大小为 $16 \times 16$ 的图像块 集, 成对地输送到预先训练的 SCNN 模型。

步骤 2: 将 SCNN 模型两个分支输出的 256 个特 征映射级联, 再与 256 维特征向量完全连接可以得到 特征映射图。

步骤 3: 将步骤 2 得到的特征映射图重叠区域块 “求平均” , 从而得到与源图像具有相同尺寸的特征 映射图。

步骤 4: 将步骤 3 得到的特征映射图中概率值大 于 0.5 的视为细节信息特征, 置为 1 ; 小于 0.5 的, 视 为非细节特征信息, 置为 0 。

步骤 5 : 将步骤 4 得到的特征映射图输入到 Softmax 分类器, 并重新按照顺序拼接可以得到二值 图。

步骤 6: 将步骤 5 得到的二值图进行滤波处理, 得到 NSST 域低频子图系数融合最终的决策图 $W$ 。

步骤 7: 将成对输入的图像块 $A_{\mathrm{L}}{ }^{\prime}$ 和 $B_{\mathrm{L}}{ }^{\prime}$ 进行拉普 拉斯分解, 分别得到 $L\left(A_{\mathrm{L}}{ }^{\prime}\right)^{l}$ 子带和 $L\left(B_{\mathrm{L}}{ }^{\prime}\right)^{l}$ 。其中, $l$ 为拉普拉斯分解层级。

步骤 8: 将步骤 6 得到的决策图 $W$ 进行高斯滤波 处理, 得到 $W$ 分解子带 $G(W)^{l}$ 。其中, 步骤 7 和步骤 8 的分解级数设置为 $\left\lfloor\log _{2} \min (h, w)\right\rfloor, w$ 和 $h$ 分别为

源图像块的空间大小， $\lfloor$ 」为向下取整。

步骤 9: 根据式(6)分别完成 $L\left(A_{\mathrm{L}}{ }^{\prime}\right)^{l}$ 子带和 $L\left(B_{\mathrm{L}}{ }^{\prime}\right)^{l}$ 子带的局部区域能量 $E\left(A_{\mathrm{L}}{ }^{\prime}\right)^{l}(x, y)$ 和 $E\left(B_{\mathrm{L}}{ }^{\prime}\right)^{l}(x, y)$ 的计算:

$$
\left\{\begin{array}{l}
E\left(A_{\mathrm{L}}{ }^{\prime}\right)^{l}(x, y)=\sum_{m} \sum_{n} L\left(A_{\mathrm{L}}{ }^{\prime}\right)^{l}(x+m, y+n)^{2} \\
E\left(B_{\mathrm{L}}{ }^{\prime}\right)^{l}(x, y)=\sum_{m} \sum_{n} L\left(B_{\mathrm{L}}\right)^{l}(x+m, y+n)^{2}
\end{array}\right.
$$

步骤 10 : 设计了基于局部相似性的测量函数 $S^{l}(x, y)$ 。其中, $S^{l}(x, y)$ 的函数值范围为 $[-1,1]$, 设置阈 值 $D$ 来自适应地调整融合模式。 $S^{l}(x, y)$ 测量函数如式 (7)表示:

$$
S^{l}(x, y)=\frac{2 \sum_{m} \sum_{n} L\left(A_{\mathrm{L}}{ }^{\prime}\right)^{l}(x+m, y+n) L\left(B_{\mathrm{L}}{ }^{\prime}\right)^{l}(x+m, y+n)}{E\left(A_{\mathrm{L}}\right)^{l}(x, y)+E\left(B_{\mathrm{L}}\right)^{l}(x, y)}
$$

步骤 11: 当相似性较大时, SCNN 模型提取的特 征信息是可靠的, 此时可以根据 SCNN 模型输出的决 策图进行低频系数融合:

$$
L\left(F_{\mathrm{L}}\right)_{s \geq D}^{l}=G(W)^{l} \cdot L\left(B_{\mathrm{L}}{ }^{\prime}\right)^{l}+\left(1-G(W)^{l}\right) \cdot L\left(B_{\mathrm{L}}{ }^{\prime}\right)^{l}
$$

步骤 12: 相似性较低时, 决策图 $W$ 是不可靠的, 此时应该选择局部区域能量较大者, 式(9)为具体的融
合模式:

$$
L\left(F_{\mathrm{L}}\right)^{\prime}(x, y)= \begin{cases}L\left(F_{\mathrm{L}}\right)_{S \geq D}^{l}(x, y) & S^{l}(x, y) \geq D \\ L\left(\mathrm{~A}_{\mathrm{L}}\right)^{l}(x, y) & S^{l}(x, y)<D \& \\ & E\left(\mathrm{~A}_{\mathrm{L}}\right)^{l}(x, y) \geq E\left(B_{\mathrm{L}}\right)^{l}(x, y) \\ & S^{l}(x, y)<D \& \\ & E\left(B_{\mathrm{L}}\right)^{l}(x, y)>E\left(A_{\mathrm{L}}\right)^{\prime}(x, y)\end{cases}
$$

步骤 13: 将融合的低频子带 $L\left(F_{\mathrm{L}}{ }^{\prime}\right)^{l}(x, y)$ 进行拉普 拉斯逆变换, 可以得到融合的 NSST 域低频子图。

\section{2 基于 APCNN 模型的高频系数融合}

经典的高频子带融合模式不能够充分利用高频 细节特征, 不仅容易导致图像的结构和空间信息大量 丢失, 而且容易引入噪声和忽略细节。

因此, 本文算法克服了需要人为设置 PCNN 模型 自由参数的难题 ${ }^{[13]}$, 根据红外和可见光图像类型的特 征分别选用了子带的标准偏差、局部区域能量和以及 局部区域可见度信息自适应地设置网络参数来估计 NSST 域高频子带系数的细节强度, 可以有效地保留高 频子带的显著特征信息, 符合人类的视觉神经感知。

对于基于 PCNN 模型的融合模式, 通常选用单个 像素的灰度值来激励 PCNN 模型。但是, 人类的视觉 系统对单个像素是不敏感的, 因此本文算法选用整个 高频子图的绝对值来激励 APCNN 模型, 即:

$$
F_{i j}=S_{i j}=\operatorname{ABS}\left(I_{\mathrm{H}}\right)
$$

链接强度 $\beta$ 可以改变链接通道权重来决定邻域神 经元的内部活动状态, 通常是通过实验来确定 $\beta$ 值。 但是根据人眼视觉系统原理, 神经元的 $\beta$ 值是随着周 围特性的变化而变化的, 因此 $\beta$ 值需要随着高频子图 的特征来自适应地调节。根据 1.2 节 APCNN 的设计, 设 $\lambda=\beta V_{\mathrm{L}}$, 则 $\alpha_{\mathrm{L}} 、 \lambda 、 V_{E}$ 以及 $\alpha_{\mathrm{e}}$ 可以分别由式(2)、式 (3)、式(4)以及式(5)表示。

式(2)的 $\sigma(S)$ 为输入图像的标准偏差。但是, 对于 红外与可见光图像类型, 局部区域能量和与局部区域 可见光越大, 表示视觉显著性区域包含了较多的边 缘、轮廓以及纹理特征。因此, 设 $\sigma(S)$ 分别为局部区 域能量和 EOL、局部区域可见度 VI 以及标准偏差 SD 来自适应地调节 APCNN 模型的自由参数, 捕捉 NSST 域高频子图更多的特征信息, 即:

$$
\sigma(S)=\mathrm{EOL}=\sum_{(u, v) \in w}(f(u, u)+f(v, v))^{2}
$$

$$
\sigma(S)=\mathrm{VI}=\frac{1}{N} \sum_{(u, v) \in \mathrm{w}}\left(\frac{1}{m_{k}}\right)^{\alpha} \cdot \frac{\left|f(u, v)-m_{k}\right|}{m_{k}}
$$




$$
\sigma(S)=\mathrm{SD}=\sqrt{\frac{\sum_{i=1}^{l} \sum_{j=1}^{l}\left(f(u, v)-m_{k}\right)^{2}}{l \cdot l}}
$$

式中: $f(u, v)$ 表示 $(u, v)$ 处的像素值; $w$ 表示尺寸为 $l \times l$ 的窗口; $m_{k}$ 表示像素平均值; $N$ 表示窗口像素数; $\alpha$ 表示常数。

NSST 域高频子带系数融合的具体流程如下所示:

步骤 1: 分别计算出 NSST 域红外高频子图 $A_{\mathrm{H}}$ 和 可见光高频子图 $B_{\mathrm{H}}$ 的绝对值 $A_{\mathrm{H}}{ }^{\mathrm{a}}$ 和 $B_{\mathrm{H}}{ }^{\mathrm{a}}$ 。

步骤 2: 将绝对值 $A_{\mathrm{H}}{ }^{\mathrm{a}}$ 和 $B_{\mathrm{H}}{ }^{a}$ 分别归一化处理, 赋 值给 $F_{i j}$ 激励 APCNN 模型。

步骤 3: 将 APCNN 模型进行参数初始化, 即:

$$
\left\{\begin{array}{l}
L_{i j}[0]=U_{i j}[0]=E_{i j}[0]=Y_{i j}[0]=0 \\
n=N_{\max }
\end{array}\right.
$$

步骤 4: 设定 EOL、VI 以及 SD 特征分别为 $\sigma(S)$ 。

根据公式(2) (5)分别计算出 APCNN 模型自适 应参数。

步骤 5: 根据 APCNN 模型的运行机制, 实现 $N_{\text {max }}$ 次迭代, EOL、VI 以及 $\mathrm{SD}$ 特征调参的点火次数分别 为: $T_{\mathrm{A}}{ }^{\mathrm{E}}\left[N_{\max }\right] 、 T_{\mathrm{A}}{ }^{\mathrm{V}}\left[N_{\text {max }}\right] 、 T_{\mathrm{A}}^{\mathrm{S}}\left[N_{\text {max }}\right] 、 T_{\mathrm{B}}^{\mathrm{E}}\left[N_{\text {max }}\right] 、 T_{\mathrm{B}}{ }^{\mathrm{V}}\left[N_{\max }\right]$ 以及 $T_{\mathrm{B}} \mathrm{S}\left[N_{\text {max }}\right]$ 。

步骤 6: 根据显著性特征调参的点火输出, 构造 加权函数:

$$
\begin{aligned}
& W_{A}^{\mathrm{E}}=\frac{T_{A}^{\mathrm{E}}}{\left(T_{A}^{\mathrm{E}}+T_{A}^{\mathrm{V}}+T_{A}^{\mathrm{S}}\right)} \quad W_{B}^{\mathrm{E}}=\frac{T_{B}^{\mathrm{E}}}{\left(T_{B}^{\mathrm{E}}+T_{B}^{\mathrm{V}}+T_{B}^{\mathrm{S}}\right)} \\
& W_{A}^{\mathrm{V}}=\frac{T_{A}^{\mathrm{V}}}{\left(T_{A}^{\mathrm{E}}+T_{A}^{\mathrm{V}}+T_{A}^{\mathrm{S}}\right)} \quad W_{B}^{V}=\frac{T_{B}^{\mathrm{V}}}{\left(T_{B}^{\mathrm{E}}+T_{B}^{\mathrm{V}}+T_{B}^{\mathrm{S}}\right)} \\
& W_{A}^{\mathrm{S}}=\frac{T_{A}^{\mathrm{S}}}{\left(T_{A}^{\mathrm{E}}+T_{A}^{\mathrm{V}}+T_{A}^{\mathrm{S}}\right)} \quad W_{B}^{S}=\frac{T_{B}^{\mathrm{S}}}{\left(T_{B}^{\mathrm{E}}+T_{B}^{\mathrm{V}}+T_{B}^{\mathrm{S}}\right)}
\end{aligned}
$$

步骤 7: 根据步骤 6 设计的加权函数, 构造红外 高频子图 $A_{\mathrm{H}}$ 和可见光高频子图 $B_{\mathrm{H}}$ 的点火映射图:

$$
\begin{aligned}
& T_{A}^{\mathrm{H}}=W_{A}^{\mathrm{E}} \cdot T_{A}^{\mathrm{E}}+W_{A}^{\mathrm{V}} \cdot T_{A}^{\mathrm{V}}+W_{A}^{\mathrm{S}} \cdot T_{A}^{\mathrm{S}} \\
& T_{B}^{\mathrm{H}}=W_{B}^{\mathrm{E}} \cdot T_{B}^{\mathrm{E}}+W_{B}^{\mathrm{V}} \cdot T_{B}^{\mathrm{V}}+W_{B}^{\mathrm{S}} \cdot T_{B}^{\mathrm{S}}
\end{aligned}
$$

步骤 8: 根据新点火图, 得到融合的高频系数:

$$
H_{\mathrm{F}}(i, j)= \begin{cases}H_{A}(i, j), & \text { if } O_{A}(i, j)>O_{B}(i, j) \\ H_{B}(i, j), & \text { if } O_{A}(i, j)<O_{B}(i, j) \\ \frac{H_{A}(i, j)+H_{B}(i, j)}{2}, & \text { if } O_{A}(i, j)=O_{B}(i, j)\end{cases}
$$

\section{4 实验结果与分析}

\section{1 实验说明}

为了验证 NSST-CNN-APCNN 算法的实用性, 选 用了大小为 $256 \times 256$ 预配准的 “森林” (场景复杂,
而目标单一）、“船”（场景单一，而目标复杂）以 及 “商店” (场景复杂, 且目标复杂) 3 组红外与可见 光图像进行融合实验, 并将 NSST-CNN-APCNN 算法 与较为先进的 5 种融合算法进行性能比较, 对比算法 分别为: 文献[14] (简称 NSCT-PCNN 算法)、文献[15] （简称 SR 算法）、文献[16]（简称 NSST 算法）、文 献[17]（简称 NSST-PAPCNN 算法）以及文献[18]（简 称 CNN 算法）。其中, 仿真环境为 2016b MATLAB, 各组源图像均进行 “归一化” 处理, 对于 NSST 变换 部分, 均选用 “maxflat” 滤波器, 分解级数为 4 , 方向 子带为 $[8,8,16,16]$ (其他参数根据文献设定）。

\section{2 融合评价}

图像融合质量评价方法主要有两个方面, 即主观 视觉评价和客观指标评价。为了可以更加客观地评价 NSST-CNN-APCNN 算法的性能, 选用了空间频率 $(\mathrm{SF}) 、$ 清晰度 $(\mathrm{SP})$ 、边缘信息传递因子 $\left(Q^{\mathrm{AB} / \mathrm{F}}\right)$ 、 结构相似模型 (SSIM) 以及视觉信息保真度 ${ }^{[19]}$ (VIFF) 5 个评价指标评估融合图像的融合质量 (指标值越大, 融合质量越高, 融合效果越好）。

第一组 “森林” 图像实验素材如图 7(a) (b)所示, 图 7(a)是可见光灰度图像, 图 7(b)是与之对应的红外 图像。而图 7(c) (h) 是不同算法对图 7(a) (b)进行图 像融合得到的融合结果灰度图像。

从图 7(c) (h) 融合结果可以看出, 上述融合算法 均可以有效地将源图像的互补信息进行融合, 既显示 了目标, 又刻画了场景。但是, 不同的融合算法捕捉 特征信息的能力也是各不相同的。NSCT-PCNN 算法 的实验结果中, 护栏纹理清晰, 但树木出现 “重影” 现象。SR 算法的实验结果中, 目标和场景层次感增 强, 但对比度严重减弱。NSST 算法和 NSST-PAPCNN 算法的实验结果中, 目标人物清晰, 但纹理信息丢失 严重。CNN 算法的实验结果中, 灰度层次感较强, 但 屋檐左侧的树木存在空间 “不连续” 现象。NSST -CNN-APCNN 算法的实验结果中, 目标和场景对比 度增强, 纹理结构特征明显, 保留了源图像较多的细 节信息。

第一组 “森林” 图像的客观质量评价指标如表 1 所示, 评价指标值归一化走势如图 8 所示。

从表 1 的客观质量评价指标可以看出, NSST -CNN-APCNN 算法的 $Q^{\mathrm{AB} / \mathrm{F}}$ 指标略低于 CNN 算法, 但 SF、SP、VIFF 以及 SSIM 指标均明显优于 5 种对 比算法。从图 8 的归一化走势可以看出, 虽然 NSST -CNN-APCNN 算法的 $Q^{\mathrm{AB} / \mathrm{F}}$ 指标略低, 但是 VIFF 指 标相比于 SR 算法提高了约 $28.62 \%$, 具有较好的视觉 保真度。综上可知, NSST-CNN-APCNN 算法捕捉边 缘信息的能力略弱于 CNN 算法, 但在活跃度、清晰 
度、保真度以及结构信息方面的保留能力优于 5 种对

比算法, 具有较优的融合性能。

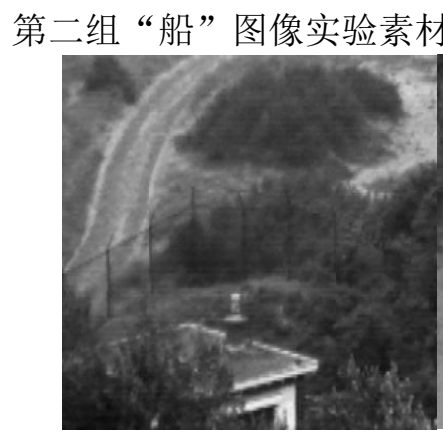

(a) 源图像 $\mathrm{A}$

(a) Source image $\mathrm{A}$

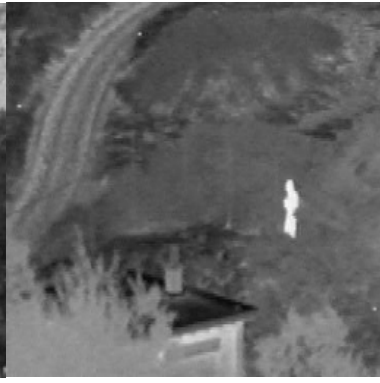

(b) 源图像 B
图 9(a)是可见光灰度图像, 图 9(b)是与之对应的红外 图像。而图 9(c) (h) 是不同算法对图 9(a) (b)进行图 像融合得到的融合结果灰度图像。

(b) Source image B

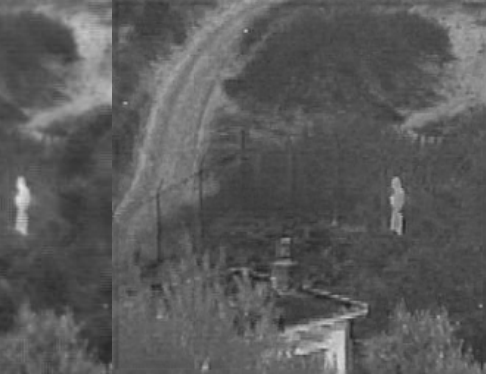

(c) NSCT-PCNN 算法

(d) SR 算法

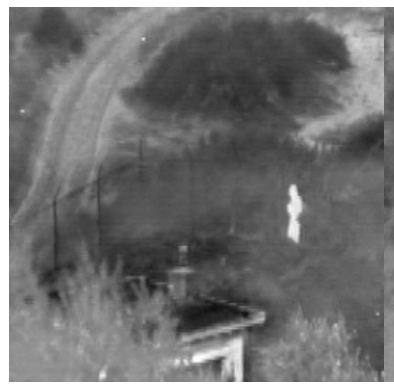

(e) NSST 算法

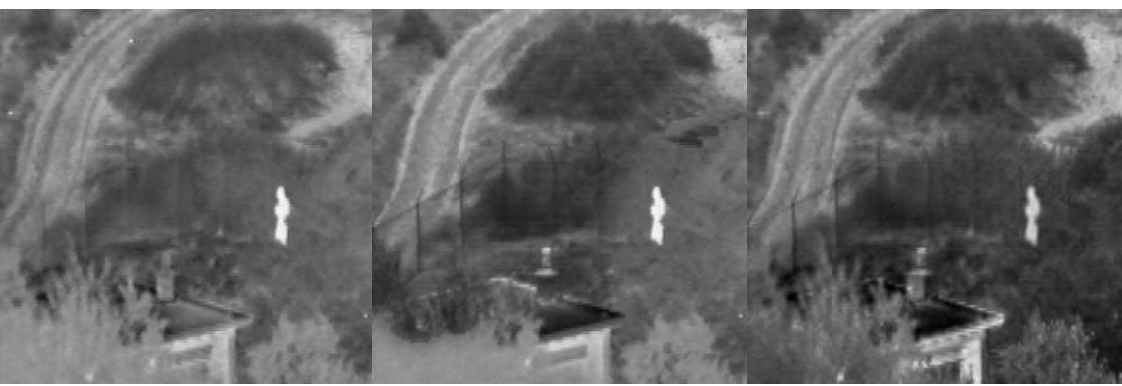

(e) NSST algorithm

(f) NSST-PAPCNN 算法

(f) NSST-PAPCNN algorithm (g) CNN 算法

(h) NSST-CNN-APCNN 算法

图 7 “森林” 图像不同算法的融合结果 Fig.7 Fusion results of different algorithms for "forest" images 表 1 “森林” 客观质量评价指标

Table 1 “forest” objective quality evaluation indicators

\begin{tabular}{ccccccc}
\hline Evaluation & NSST-CNN-APCNN & CNN & NSST-PAPCNN & NSST & SR & NSCT-PCNN \\
\hline SF & 5.5814 & 5.0345 & 5.0757 & 5.0099 & 5.5460 & 4.9441 \\
SP & 2.4499 & 2.2036 & 2.2656 & 2.1806 & 2.3631 & 2.1247 \\
$Q^{\mathrm{AB} / F}$ & 0.3921 & 0.5341 & 0.3958 & 0.4182 & 0.3933 & 0.3371 \\
VIFF & 0.4512 & 0.4497 & 0.4492 & 0.4497 & 0.3508 & 0.3780 \\
SSIM & 1.1871 & 1.1324 & 1.1764 & 1.1653 & 1.1658 & 1.1558 \\
\hline
\end{tabular}

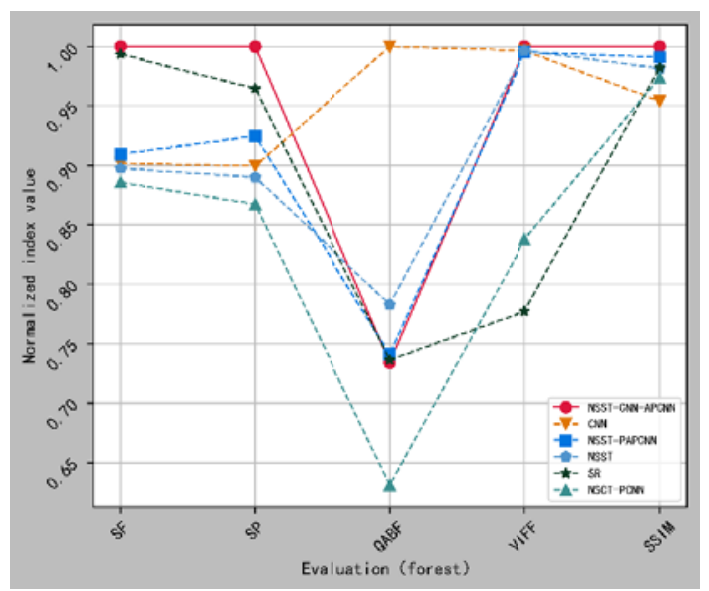

图 8 “森林” 指标值归一化走势图

Fig. 8 “forest” indicator value normalization chart 从表 2 的客观质量评价指标可以看出, NSST
-CNN-APCNN 算法的 SSIM 指标略弱于 SR 算法, 说 明其空间结构特征信息的捕捉能力弱于 SR 算法。但 是，NSST-CNN-APCNN 算法的其他客观质量评价指 标均明显优于 5 种对比算法, 可以提高观察者对目标 和场景的感知能力, 有助于目标跟踪和检测识别的实 际应用。从图 10 的归一化走势可以看出, NSST -CNN-APCNN 算法、CNN 算法以及 NSST-PAPCNN 算法的走势略为一致, NSST 算法、SR 算法以及 NSCT-PCNN 算法的走势略为一致, 且前三者的 VIFF 指标明显高于后三者, 刻画了源图像更深层面的保真 度和结构特征信息。另外, NSST-CNN-APCNN 算法 除了 SSIM 指标，其他客观质量评价指标均为 “最优 值” ，且 VIFF 指标相比于 NSCT-PCNN 算法提高了 约 $71.44 \%$ ，融合效果高度符合人眼视觉效果。 


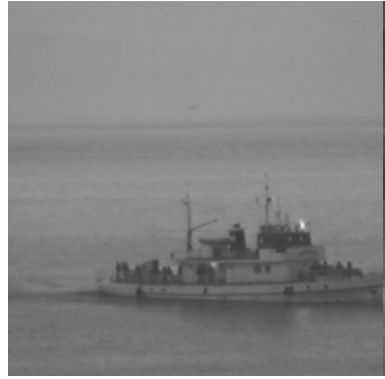

(a) 源图像 A

(a) Source image $\mathrm{A}$

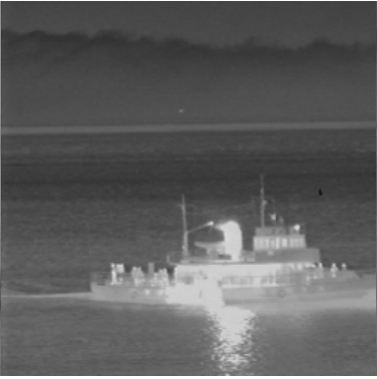

(b) 源图像 B

(b) Source image B

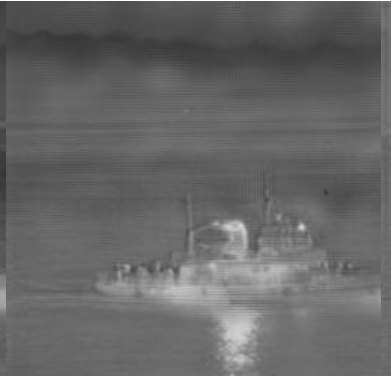

(c) NSCT-PCNN 算法

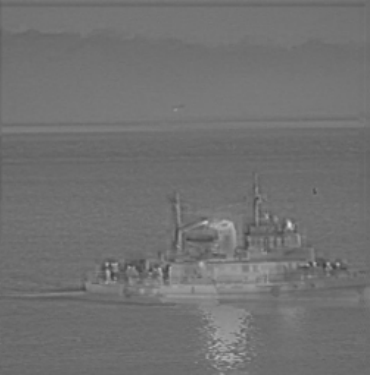

(d) SR 算法

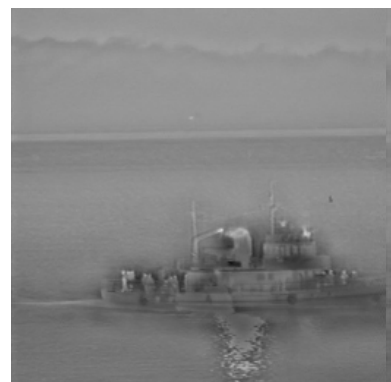

(e) NSST 算法

\begin{abstract}
(f) NSST-PAPCNN 算法
\end{abstract}
(c) NSCT-PCNN algorithm

(d) SR algorithm

(e) NSST algorithm

(f) NSST-PAPCNN algorithm

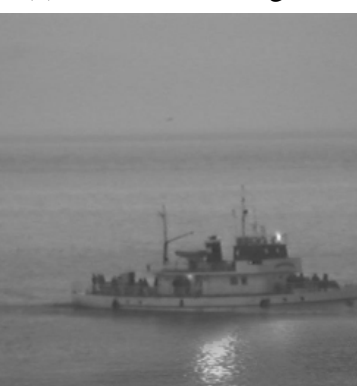

(g) CNN 算法

(g) CNN algorithm

图 9 “船” 图像不同算法的融合结果 Fig.9 Fusion results of different algorithms for "boat” images 表 2 “船” 客观质量评价指标

Table 2 "forest" objective quality evaluation indicators

\begin{tabular}{ccccccc}
\hline Evaluation & NSST-CNN-APCNN & CNN & NSST-PAPCNN & NSST & SR & NSCT-PCNN \\
\hline SF & 3.8706 & 2.9689 & 3.1644 & 3.3424 & 3.3524 & 3.3428 \\
SP & 1.3447 & 1.0209 & 1.2068 & 1.2223 & 1.3223 & 1.2228 \\
$Q^{\mathrm{AB} / F}$ & 0.4747 & 0.4647 & 0.3860 & 0.4183 & 0.4675 & 0.3577 \\
VIFF & 0.4485 & 0.4477 & 0.4431 & 0.3103 & 0.2803 & 0.2616 \\
SSIM & 1.3898 & 1.3685 & 1.3010 & 1.3522 & 1.4321 & 1.2090 \\
\hline
\end{tabular}

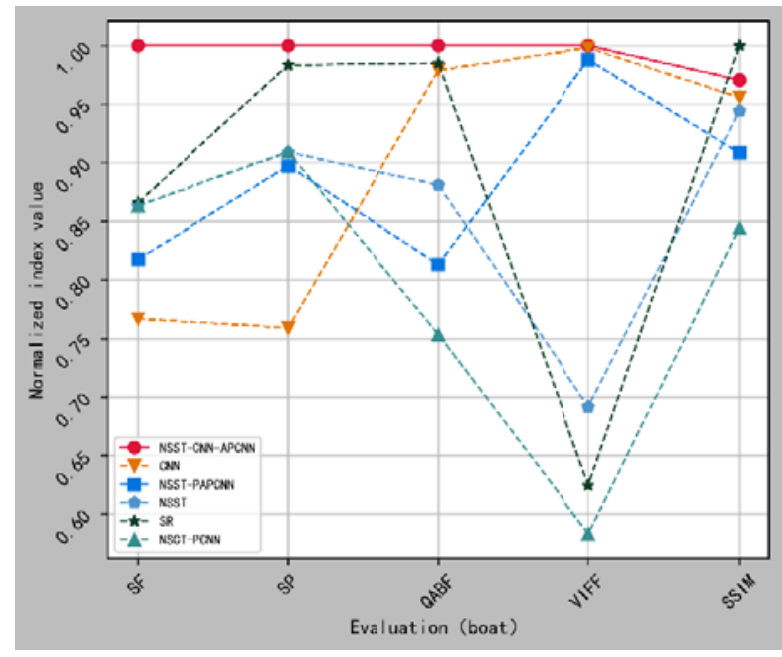

图 10 “船” 指标值归一化走势图

Fig. 10 "boat" indicator value normalization chart

第 3 组 “商店” 图像实验素材如图 11(a) (b)所 示, 图 11(a)是可见光灰度图像, 图 11(b)是与之对应 的红外图像。而图 11(c) (h) 是不同算法对图 11(a) (b)进行图像融合得到的融合结果灰度图像。
从图 11(c) (h) 融合结果可以看出, NSCT-PCNN 算法的实验结果中, 物体边缘轮廓模糊, 丢失了部分 亮度信息, 分辨率较差。SR 算法的实验结果中, 目 标和场景纹理清晰, 但亮度信息丢失明显。NSST 算 法的实验结果中, 物体边缘过度平滑, 且出现了重影 干扰。CNN 算法的实验结果中, 几乎丢失了全部的可 见光信息, 不适合用于目标复杂且场景复杂类型的图 像融合。NSST-PAPCNN 算法和 NSST-CNN-APCNN 算法的实验结果中, 物体连续性较好, 细节刻画深刻, 且灰度、亮度以及锐利度比较贴近源图像。但是, NSST-PAPCNN 算法的目标均存在 “虚影”和“重影” 的现象, 使得人眼甄别困难。因此, NSST -CNN-APCNN 算法的融合结果可以更好地分配可见 光和红外信息, 有效地消除了 “伪影” , 保留了较好 的细节信息。

第 3 组 “商店” 图像的客观质量评价指标如表 3 所示, 评价指标值归一化走势如图 12 所示。 


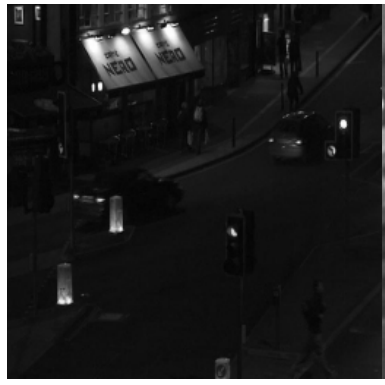

(a) 源图像 A

(a) Source image $\mathrm{A}$

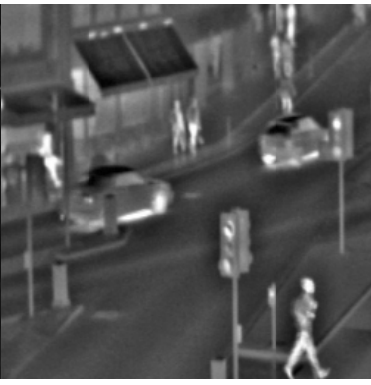

(b) 源图像 B

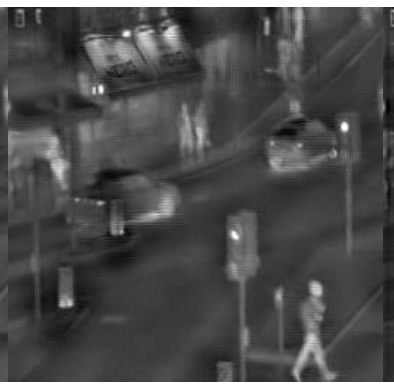

(c) NSCT-PCNN 算法

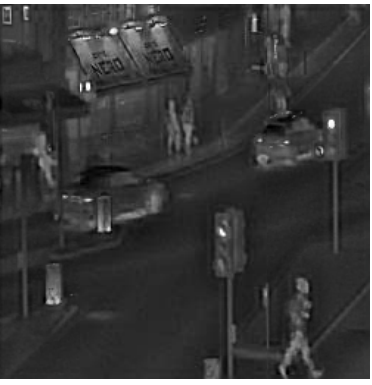

(d) SR 算法

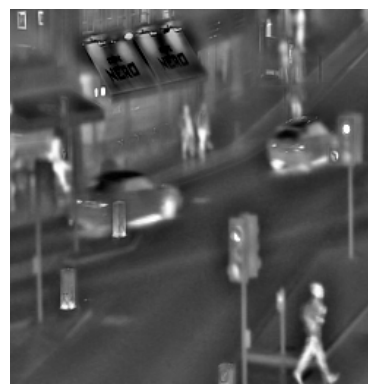

(e) NSST 算法

(b) Source image B

(c) NSCT-PCNN algorithm

(d) SR algorithm

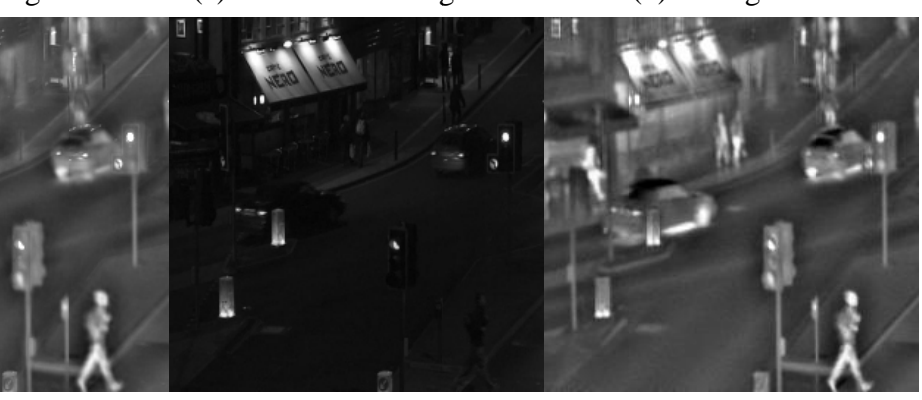

(f) NSST-PAPCNN 算法

(g) CNN 算法

(h) NSST-CNN-APCNN 算法

(e) NSST algorithm

(f) NSST-PAPCNN algorithm

(g) CNN algorithm

(h) NSST-CNN-APCNN algorithm

图 11 “商店” 图像不同算法的融合结果

Fig.11 Fusion results of different algorithms for " store " images

表 3 “商店” 客观质量评价指标

Table 3

\begin{tabular}{ccccccc}
\hline Evaluation & NSST-CNN-APCNN & CNN & NSST-PAPCNN & NSST & SR & NSCT-PCNN \\
\hline SF & 6.2093 & 4.1279 & 2.8780 & 5.9989 & 5.7597 & 5.5744 \\
SP & 2.4960 & 1.3461 & 2.3243 & 2.4099 & 2.3643 & 2.1847 \\
$Q^{\mathrm{AB} / \mathrm{F}}$ & 0.5651 & 0.3712 & 0.5358 & 0.6263 & 0.4087 & 0.3477 \\
VIFF & 0.7144 & 0.7144 & 0.6510 & 0.7111 & 0.4484 & 0.4228 \\
SSIM & 1.0878 & 1.0983 & 1.0425 & 1.0983 & 1.0381 & 0.9837 \\
\hline
\end{tabular}

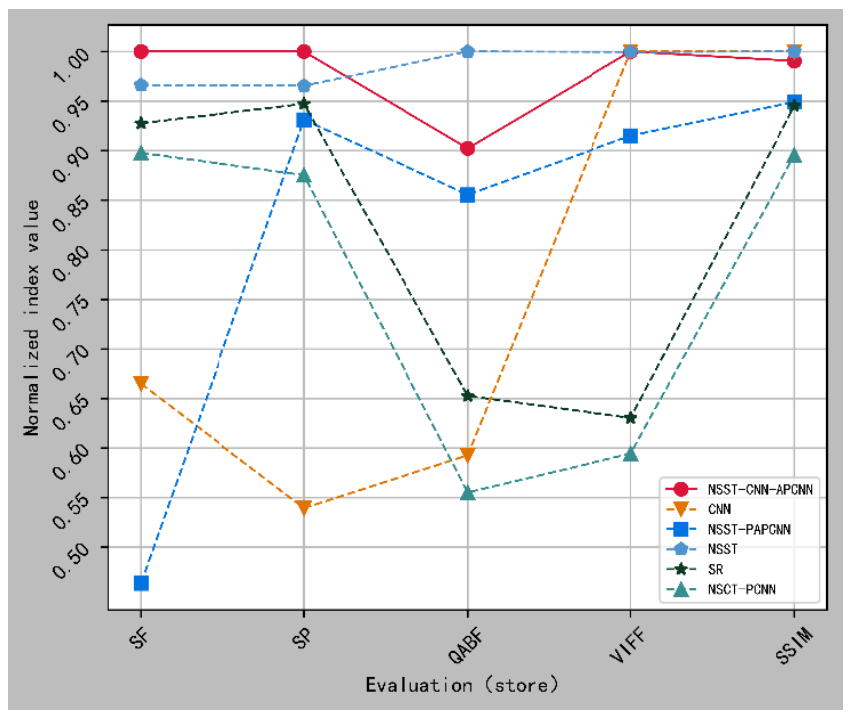

图 12 “商店” 指标值归一化走势

Fig.12 "Store" indicator value normalization chart

从表 3 的客观质量评价指标可以看出, NSST -CNN-APCNN 算法的 $Q^{\mathrm{AB} / \mathrm{F}}$ 指标和 SSIM 指标略弱于
NSST 算法和 CNN 算法, 说明 NSST-CNN-APCNN 算法遗失了少数的边缘细节信息和深度结构特征。但 是, NSST-CNN-APCNN 算法的 SF、SP 以及 VIFF 指 标依旧明显优于 5 种对比算法, 能够较好地改善图像 的分辨率、增强图像的清晰度以及提高图像的保真 度。从图 12 的归一化走势可以看出, NSST-CNN-APCNN 算法和 NSST 算法的各项客观评 价指标均明显优于其他 4 种对比算法。另外, NSST-CNN-APCNN 算法在 $Q^{\mathrm{AB} / \mathrm{F}}$ 指标和 SSIM 指标略 弱于 NSST 算法, 但从图 11(e)中可以看出, NSST 算 法的融合效果存在 “振铃效应”, 从主观视觉评价和 客观指标评价综合分析来看, NSST-CNN-APCNN 算 法捕捉了更多的清晰度、相似性以及边缘纹理等细节 信息, 具有较好的可识别度。

\section{5 结束语}

NSST-CNN-APCNN 算法将 NSST 变换、卷积神 
经网络以及参数自适应的 PCNN 神经元模型相互结 合, 解决了 NSST 分解低频子带融合模式给定的问题, 同时又避免了手动设置脉冲耦合神经网络 (PCNN) 参数的困难。为了提取到源图像更加丰富的信息, NSST-CNN-APCNN 算法对于 NSST 分解的低频和高 频系数分别进行了更加细致的处理, 其将低频子带进 行了拉普拉斯分解, 将 SCNN 模型输出的决策图进行 了高斯滤波分解, 最终在每个分解层级上完成了低频 系数的融合。对于高频子带的融合, 其分别使用了方 差、局部区域能量以及可见度特征来激励参数自适应 PCNN 神经元模型。综合分析实验结果可知, 在主观 和客观评价方面, NSST-CNN-APCNN 算法均取得了 较好的融合结果, 较好地低刻画了源图像的结构、纹 理以及相似性等特征，有效地抑制了 “伪影” 和 “失 真” 状况的出现, 并且在 “森林” 图像实验中, 融合 图像的 SF 指标、SP 指标、VIFF 指标以及 SSIM 指标 相比于对比算法中最高的分别提高了约 $0.64 \%$ 、 $3.67 \% 、 0.33 \%$ 以及 $0.91 \%$ 。在 “船” 图像实验中, 融 合图像的 SF 指标、SP 指标、 $Q^{\mathrm{AB} / \mathrm{F}}$ 指标、VIFF 指标 以及 SSIM 指标相比于对比算法中最高的分别提高了 约 $15.26 \% 、 1.69 \% 、 1.54 \% 、 0.18 \%$ 以及 $1.56 \%$ 。在 “商 店” 图像实验中, 融合图像的 SF 指标和 SP 指标相比 于对比算法中最高的分别提高了约 $3.51 \%$ 和 $3.57 \%$ 。 且 VIFF 指标和最高的指标一样。综上可以看出, NSST-CNN-APCNN 算法可以在保证其他指标减少较 少的情况下提高图像的 SF 指标、SP 指标以及 VIFF 指标，具有刻画源图像更深层面几何结构的能力。

\section{参考文献:}

[1] 杨勇, 万伟国, 黄淑英, 等. 稀疏表示和非下采样 Shearlet 变换相结合 的多聚焦图像融合 [J]. 小型微型计算机系统, 2017, 38(2): 386-392.

YANG Yong, WAN Weiguo, HUANG Shuying, et al. Multi focus image fusion based on sparse representation and Nonsubsampled shearlet transform[J]. Minicomputer System, 2017, 38(2): 386-392.

[2] 李娇, 杨艳春, 党建武, 等. NSST 与引导滤波相结合的多聚焦图像融 合算法[J]. 哈尔滨工业大学学报, 2018, 50(11): 145-152.

LI Jiao, YANG Yanchun, DANG Jianwu, et al. Multi focus image fusion algorithm based on NSST and guided filtering[J]. Journal of Harbin Institute of Technology, 2018, 50(11): 145-152.

[3] LIU Y , CHEN X, CHENG J , et al. Infrared and visible image fusion with convolutional neural networks[J]. International Journal of Wavelets, Multiresolution and Information Processing, 2018: S0219691318500182.

[4] DU Chaoben, GAO Shesheng. Image segmentation-based multi-focus image fusion through multi-scale convolutional neural network[J]. IEEE Access, 2017, 5: 15750-15761.

[5] 朱芳, 刘卫. 基于自适应 PCNN 模型的四元数小波域图像融合算法 [J]. 红外技术, 2018, 40(7): 660-667.

ZHU Fang, LIU Wei. Quaternion wavelet domain image fusion algorithm based on adaptive PCNN model [J]. Infrared Technology, 2018, 40(7):
660-667.

[6] CHEN Y, PARK S K, MA Y, et al. A new automatic parameter setting method of a simplified PCNN for image segmentation[J]. IEEE Trans. Neural Netw., 2011, 22(6): 880-892.

[7] KONG W W. Multi-sensor image fusion based on NSST domain I2CM[J]. Electronics Letters, 2013, 49(13): 802-803.

[8] YANG Y, YANG M, HUANG S, et al. Robust sparse representation combined with adaptive PCNN for multifocusimage fusion[J]. IEEE Access, 2018, 6: 20138-20151.

[9] Ouerghi H, Mourali O, Ezzeddine Z. Non-subsampled shearlet transform based MRI and PET brain image fusion using simplified pulse coupled neural network and weight local features in YIQ color space[J]. IET Image Processing, 2018, 12(10): 1873-1880.

[10] CHEN Y, PARK S, MA Y, et al. A new automatic parameter setting method of a simplified PCNN for image segmentation[J]. IEEE Transactions on Neural Networks, 2011, 22(6): 880-892.

[11] YU Y, GONG Z, WANG C, et al. An unsupervised convolutional feature fusion network for deep representation of remote sensing images[J]. IEEE Geoscience and Remote Sensing Letters, 2018, 15(1): 23-27.

[12] Zagoruyko S, Komodakis N. Learning to compare Image patches via convolutional neural networks[C]//Computer Vision and Pattern Recognitionof IEEE, 2015: 4353-4361.

[13] 戴进墩, 刘亚东, 毛先胤, 等. 基于 FDST 和双通道 PCNN 的红外与 可见光图像融合 [J]. 红外与激光工程, 2019, 48(2): 67-74.

DAI Jindun, LIU Yadong, MAO Xianyin, et al. Infrared and visible image fusion based on fdst and dual channel PCNN[J]. Infrared and Laser Engineering, 2019, 48(2): 67-74.

[14] 郝文超, 贾年. NSCT 域内基于自适应 PCNN 的红外与可见光图像融 合方法[J]. 西华大学学报: 自然科学版, 2014, 33(3): 11-14.

HAO Wenchao, JIA Nian. Infrared and visible image fusion method based on adaptive PCNN in NSCT domain[J]. Journal of Xihua University: Natural Science Edition, 2014, 33(3): 11-14.

[15] 张生伟, 李伟, 赵雪景. 一种基于稀疏表示的可见光与红外图像融合 方法[J]. 电光与控制, 2017, 24(6): 47-52.

ZHANG Shengwei, LI Wei, ZHAO Xuejing. A visible and infrared image fusion method based on sparse representation[J]. Electrooptics and Control, 2017, 24(6): 47-52.

[16] GAO Guorong, XU Luping, FENG Dongzhu. Multi-focus image fusion based on non-subsampled shearlet transform[J]. IET Image Processing, 2013, 7(6): 633-639.

[17] YIN Ming, LIU Xiaoning, LIU Yu, et al. Medical image fusion with parameter-adaptive pulse coupled neural network in nonsubsampled shearlet transform domain[J]. IEEE Transactions on Instrumentation and Measurement, 2019, 68(1): 49-64.

[18] 梅礼晔, 郭晓鹏, 张俊华, 等. 基于空间金字塔池化的深度卷积神经 网络多聚焦图像融合 $[\mathrm{J}]$. 云南大学学报: 自然科学版, 2019, 41(1): 18-27.

MEI Liye, GUO Xiaopeng, ZHANG Junhua, et al. Multi focus image fusion using deep convolution neural network based on spatial pyramid pooling[J]. Journal of Yunnan University: Natural Science Edition, 2019, 41(1): 18-27.

[19] Sheikh H R, Bovik A C. Image information and visual quality[J]. IEEE Transactions on Image Processing, 2006, 15(2): 430-444. 